\title{
To Predict the Waterfastness Rate of Foil Print Applying Artificial Neural Network
}

\author{
Mahasweta Mandal \\ Printing Engineering Department \\ Jadavpur University, Kolkata, India
}

\author{
Swati Bandyopadhyay \\ Printing Engineering Department \\ Jadavpur University, Kolkata, India
}

\begin{abstract}
The aim of this present study to evaluate the print quality by the waterfastness properties of foil prints which are exposed to water or rain. Waterfastness is an important property for any kind of packaging products, specially for food and medicine packages for assessing their print stability. The fastness properties of prints can be described in terms of print durability and image stability. Moreover, the poor fastness properties of prints will affect the product sale adversely. Little work has been done to study the fastness properties of printed films and foils. This study has preferred to take the blister foils as samples which are printed by gravure process as the blister foil has extensive usage in food and medicine packaging. Water immersion method and water spray methods both are used to study the water fastness of magenta ink on foil and the results were found to be similar. The evaluation is carried out by the measured spectral curves and colorimetric values before and after exposure using the oceanographic spectroradiometer (DH2000BAL) device. A significant change in reflectance in the blue and red regions with time indicates the fading of magenta print with time. This article has proposed a newly approach based on artificial neural network (ANN) model to determine the waterfastness rate of foil prints with variation of time. A comparative analysis is also made between the ANN model and regression model. However, the artificial neural network (ANN) has given a bit more excellent prediction than regression. In the context of prediction of waterfastness, the artificial neural network (ANN) model has given optimal results with the MSE 1.2409and a correlation coefficient of 0.9990 ..
\end{abstract}

\section{General Terms}

Artificial Neural Networks, Prediction

\section{Keywords}

Waterfastness, Spectral data, ANN, CIE Lab, Gravure printing

\section{INTRODUCTION}

The color or the printed image can be affected due to light, water or moisture. The waterfastness property of prints is the ability of the print to retain its color strength and fading resistance upon exposure to water. Prints with poor waterfastness have a negative effect for sellers of various goods. In case of food or medicine products which may be kept in deep freeze, it is required that the print surface does not change its color or fades away during long term water exposure. The durability of color quality plays an important role on packaging when they are exposed to the rain or water for a long period of time. Blister foil is one of the most important packaging media which is immensely used in food and medicine packaging due to its inertness and non-reactivity properties. Prints are taken in Gravure printing process that is widely used in food and medicine package printing. This present article will investigate the waterfastness performance of the printed foils. The temperature and relative humidity can affect the water restistance properties of the prints. Different models may demonstrate the waterfastness properties of prints. It represents either hypothetical mechanism or can be empirically derived during laboratory experiments.

\subsection{Related Work}

The subject of image stability has become considerably more complex in recent years. Appearance is the prime factor and most impressive product message to attract the customers' attention. So, the appearance of the product with time-its light fastness and water fastness - may also be crucial. K. Vikman [1,2] has carried out a praiseworthy work to investigate the effects of the coating polymer system on the water fastness of soluble dyes on kaolin coated papers where the changes in color coordinates, color difference, physical properties are analyzed. Moreover, FTIR and Raman spectra is also evaluated to understand the effect of the chemical interactions on water fastness and analyzed by applying principal component analysis (PCA) and projection to latent structures (PLS). The water solubility of dyes leads to a cause of poor image water fastness. Therefore, the previous researches have suggested an approach to improve waterfastness attaching dyes chemically to the substrate surface during printing by coating the paper with silica pigments, poly vinyl alcohol binders (PVA),kaolin and cationic polymer additives[3,4,5,]. Fryberg et. al [6] have considered the dyes as the most important element of an ink contributing to image stability. Another study has taken attention focusing on the paper coating formulation to improve the water fastness property using an acrylic/nano silver resin in the ink's formulation [7]. The evaluation of washfastness test is carried out to investigate the effect of reactive dye structure and the type of penetrant in ink formulation on different kinds of paper printing [8]. M. Stankovská et al. $[9,10,11]$ have investigated the print quality which can be influenced by the surface sizing papers, coating color or pigment and binders. The two most critical elements for the water fastness of the prints are the ink formulation and the composition of the receiving media which are described in details [12]. The study has addressed poor water fastness due to dye dissolve when there is no strong binding force holding the dye to the receiving medium. Therefore, the study has suggested using the cationic latex particles that can complex with anionic dyes to improve the water fastness. K. Kasahara[13] has taken attention to introduce a new drying technology for microporous glossy paper in inkjet printing in which paper has a good waterfastness. The color degradation has been analyzed with respect to time and temperature applying the first order kinetic model [14].

In recent days, many of the praiseworthy studies have applied artificial neural network as a new intelligence technology for data analysis. Few researches have shown interest to predict the lightfastness and wash fastness of fabric samples. An 
artificial neural network model has been developed to estimate the color value like CIELab, $\mathrm{C}^{*}, \mathrm{~h}^{\circ}$ and wash fastness values of nylon [15]. Previous studies have determined the accurate reaction rate applying feed forward neural network method [16-20] or used as a tool for calculating model $[21,22]$. Few studies have predicted delta $\mathrm{E}$ from the measured color coordinate of before and after dehydration process to establish the color change of fruit or pumpkin $[23,24]$. Several studies have applied ANN model to predict the shelf life of any product which is considered as the length of time given before a product is unsuitable for sale, use, or consumption[25,26]. The ANN model is developed to estimate the lightfastness characteristics of foil prints [27].

Much of the previous researches have focused on the waterfastness properties of paper substrate, coating formulation or ink. Little or almost no work has been obtained on the waterfastness data on foil prints. But foil is an excellent media for food and medicine items. This paper introduces a new approach based on ANN to study the waterfastness properties of blister foil prints. In this study, the fading rate of printed foil samples is predicted using ANN model. The model is useful to correlate the results with potential fading occurring in a natural environment for a long period of time. A comparative study between an optimal ANN model and kinetic models will also be discussed.

\section{OBJECTIVE}

An object of the present study is to assess the water fastness properties of foil prints with time. In this study, the impact of water on printed foils has been investigated. This study has focused on the assessment of the waterfastness performance of printed blister foils based on the experimental data using artificial neural network (ANN) technique. This artificial neural network (ANN) model has been developed to predict the degradation of image quality due to water exposure at different time interval.

\section{EXPERIMENTAL MATERIAL AND METHODS}

This study has concentrated on the waterfastness characteristics of blister foil prints as it is one of the most important packaging media. It also enhances the longevity of the food and medicine than if it is wrapped in plastic. The advantageous features of gravure printing make it a very promising process for food and medicine package printing industry. Therefore, the testing is carried out on the blister foil substrate which is printed by gravure printing press. The printing press consists of an electronically engraved printing cylinder, blower, and heater. The drying temperature has been fixed at $50^{\circ}-60^{\circ} \mathrm{C}$ for the printing. The experiment is done on $100 \%$ solid patch with screen frequency 152 lines per inch. Solvent based Magenta foil ink is chosen to print the solid color patch at $60 \mathrm{~meter} / \mathrm{min}$ speed and this kind of liquid ink is specifically utilized for gravure printing on blister foil substrate. All the samples are printed at same speed and pressure. The ambient temperature and humidity are $17 \pm 3^{\circ} \mathrm{C}$ and $35 \pm 5 \%$ inside the press. The experiment is carried out with multiple samples and five samples are taken for each exposure to check the repeatability.

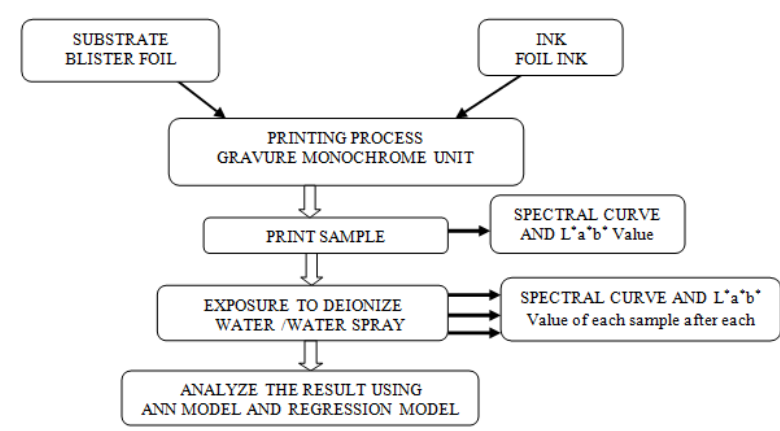

Figure 1: Workflow diagram of the Waterfastness study

\subsection{Waterfastness Test}

Water immersion and Water spray test [31] are used to evaluate the waterfastness properties of the foil prints. In water immersion test, the foil printed samples are immersed into a pan full of deionized water at room temperature. Then the specimens are pulled out of the water after different time interval, and it is allowed to dry after blotting off the excess water. This method is advantageous as all the printed samples are uniformly exposed to water. In water spray method, the printed samples are exposed to water shower at $45^{\circ}$ angle with same speed and pressure. Also the samples are placed at same distance. The drying process is same like water immersion test. The waterfastness test is performed in the absence of light. Figure 1 describes the workflow of the water fastness study for magenta foil prints.

\subsection{Measurement}

The present study has carried out a spectroscopic measurement by using Ocean Optic Spectrometer (DH2000BAL) with Tungsten Halogen and Deuterium lightsource. This device helps to get spectral data which can be used to calculate CIE tristimulus_values. CIE chromaticity coordinates and luminosity can then be calculated. After print, the spectrophotometric curves and the $L^{*}, a^{*}$ and $b^{*}$ values are recorded using Ocean Optic Spectrometer (DH2000BAL) so that the values of reference sample are stored to understand the changes after exposure. After the each exposure to water, the spectrophotometric curves of the exposed samples at different time intervals are collected using ocean optic spectroradiometer at $2^{\circ}$ standard observer. For each sample, five readings are taken and average of five readings is calculated, rotating the sample between measurements. So, all the data are collected for unexposed i.e reference prints and exposed prints to assess the waterfastness properties of prints. Moreover, the measurement procedure is completed at $17^{\circ} \mathrm{C}$ $23^{\circ} \mathrm{C}$ and in the absence of light. The five individual datasets have been prepared for the development of the ANN model to check the performance accuracy of the model. Exposure time intervals are considered as the independent variables for the experiment and the reflectance values of the samples are the dependent variables. The color change of the patches before and after water exposure is evaluated by CIELAB 2000[30] color difference formula which is shown as a measure of water fastness. The color coordinate values $\mathrm{L}^{*}, \mathrm{a}^{*}$ and $\mathrm{b}^{*}$ represent lightness, red to green and yellow to blue respectively. 


\subsection{Development of Artificial Neural Network}

Artificial neural network (ANN) is a mathematical modeling tool which has been preferred extensively in modeling complex real problems. The basic information of the neural network has been described in details to understand it as a computational tool $[28,29]$. Moreover, it has demonstrated the various types ANN, design method of ANN, different types of learning rules and the need for this computational method in the various kind of research field. In addition, the fundamental concept of selecting the data, pre-processing and balancing the data, representing the input/output pattern are presented to design a neural network. In proposed model, a multilayer feedforward network has been used for modeling to predict the waterfastness behavior of printed blister foil over the time. The general scheme of the proposed ANN network is illustrated in Figure 2. The advantage of using this neural model over simpler parametric models is its ability to approximate any input/output map, the increased flexibility and reduction in assumptions of the model. The structure of ANN is oriented with an input layer, one or more hidden layer, and an output layer. The input layer consists with input variables. One or more hidden neurons in hidden layer are utilized to capture the nonlinearity in the network. In this study, the neural network has been allowed to train using different number of nodes in the hidden layer. Finally, the output of the last hidden layer is given to the output layer. The hidden layer maps the input pattern with output pattern through a series of interconnected weights to achieve optimal net. The dependent variables are applied as output parameters in output layer.

In this study, a multilayer feed-forward artificial neural network is chosen to design the artificial neural networks (ANN) model. In this analysis, the Neural Network Toolbox of MATLAB (Mathwork, 2011) software is used to develop the proposed ANN model $[31,32]$. The input parameters of neural networks are wavelength and different time interval and the output of the system is the reflectance spectra at exposure time. In this ANN model, the used activation function between input layer and the single hidden layer is hyperbolic tangent which is defined in Eq. (1) as this activation function minimizes the calculated mean squared error values (Eq.2) than the sigmoid function and a linear function has been kept in output layer.

$$
\tanh =\frac{e^{x}-e^{-x}}{e^{x}+e^{-x}}
$$

In order to find best networks, the training data set of the proposed ANN model has been divided into three subsets including the training set, cross-validation and test data set. The training set (70\% of the data) is used to train the network, $15 \%$ of the data used as cross validation which are applied to monitor the neural network performance during training and the test set is used to verify the accuracy of the network and compare the performances of various network structures [24]. The proposed ANN model is trained by Backpropagation Levenberg-Marquardt (BP) algorithm which helps to minimize the error during neural network training and obtain the optimum value of network bias and weights. Training of the network is performed with the function 'trainlm' that is an iterative method which adjusts the connection weight and bias values according to LevenbergMarquardt (LM) algorithm. The training process is conducted for 1000 epochs. The training process has been performed till the minimum mean square error (MSE) between targets and outputs is achieved. The trained sets are utilized to predict the waterfastness properties of different unknown times which are not previously exposed to an artificial neural network. The criterion for selection of the optimum ANN structure is the MSE of the test data as well as the correlation coefficient $\left(R^{2}\right)$. The equation of mean square error (MSE) used as a criterion for the training error is determined in Eq. (2).

$M S E=\frac{1}{P} \sum_{p=1}^{P}\left(D_{p}-O_{p}\right)^{2}$

Where $\mathrm{P}$ is the total number of training data and $\mathrm{D}_{\mathrm{P}}$ is the desired outputs and $\mathrm{O}_{\mathrm{p}}$ is calculated outputs for the pth training data. The most important factors must be considered in order to design successful neural network model is number of hidden layers and number of neurons in hidden layers.

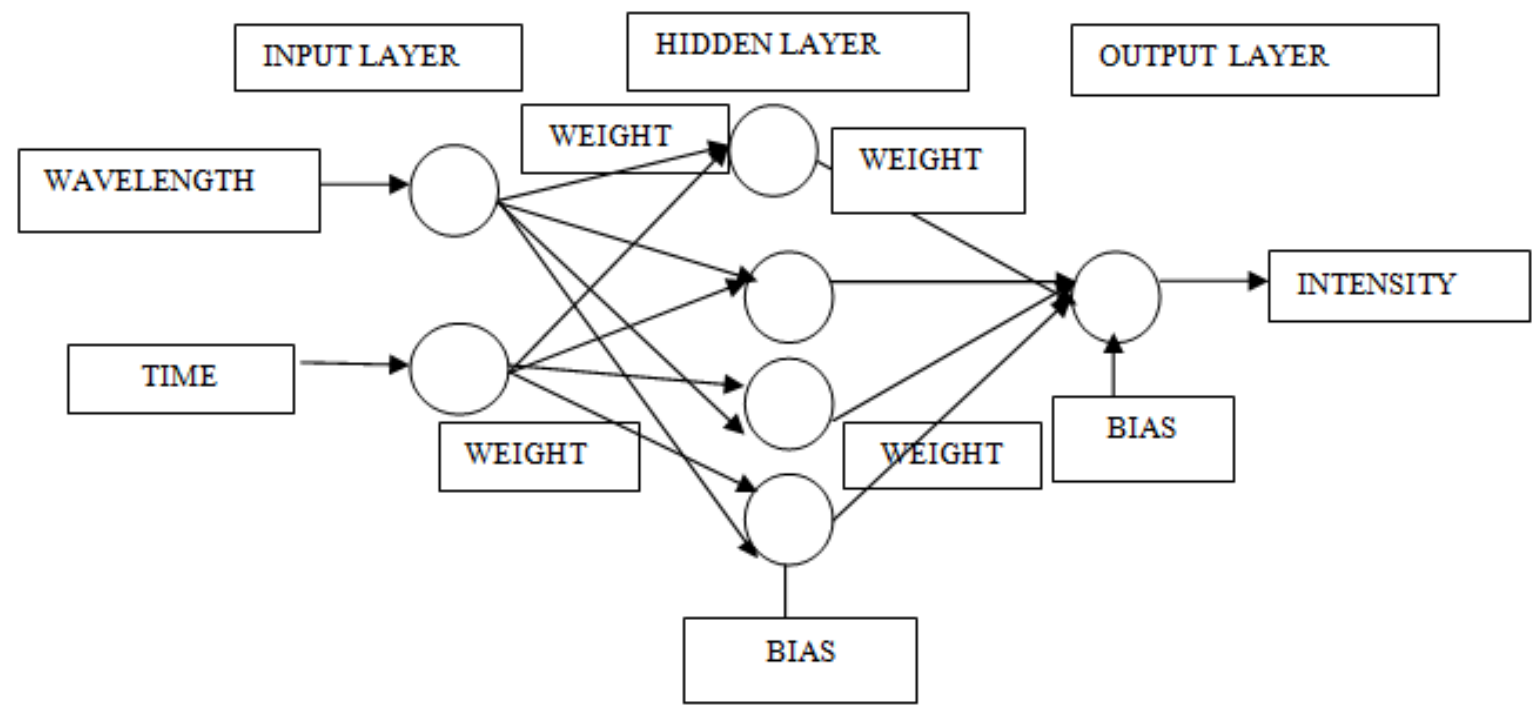

Figure 2: The architecture of the proposed feed-forward ANN 


\section{RESULTS AND DISCUSSION}

\subsection{Effect of Light Exposure on Spectral Distribution and Colorimetric Properties of Prints}

In this study, the spectrums of the printed foil before and after each exposure to water have been illustrated in Figure 3. Here the fading behavior is demonstrated by the spectral analysis as the color of the print samples is perceived by the reflectance of the samples in the visible range of spectrum. It has been observed from Figure 3 that there is a sharp increase in reflectance in the blue zone and red zone which is clearly indicating the fading with time.

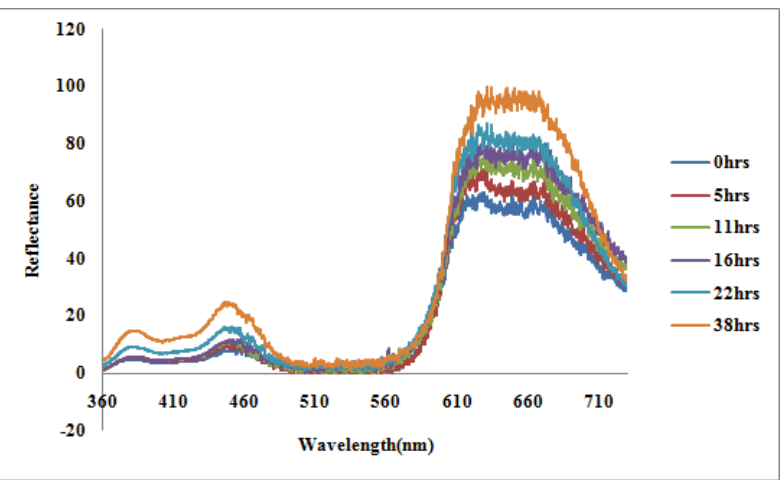

Figure 3: Spectral data of fading on magenta print on blister foi

Degradation due to water exposure can also be demonstrated with the lightness and chroma value. The variation of lightness $\mathrm{L}$ and chroma at different time are presented in the Figure 4 and Figure 5 respectively. It is visible in Figure 4 that the $\mathrm{L}$ values of the print are increasing with time which clearly indicates the fading of color. Figure 5 shows the changes in chroma value which is reduced with time.

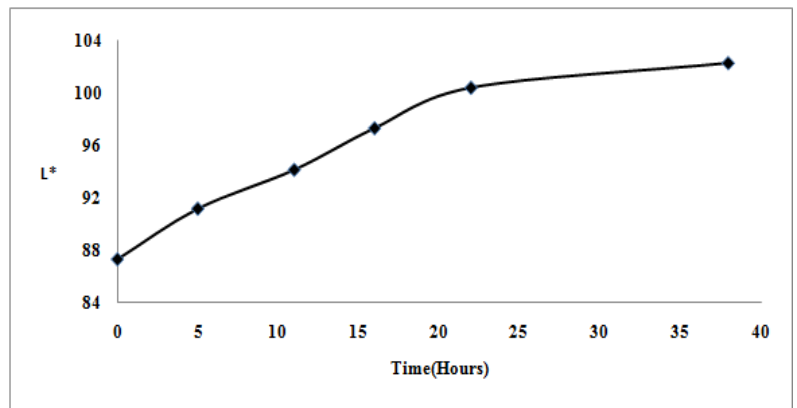

Figure 4: Changes in $L$ value of Printed blister foil

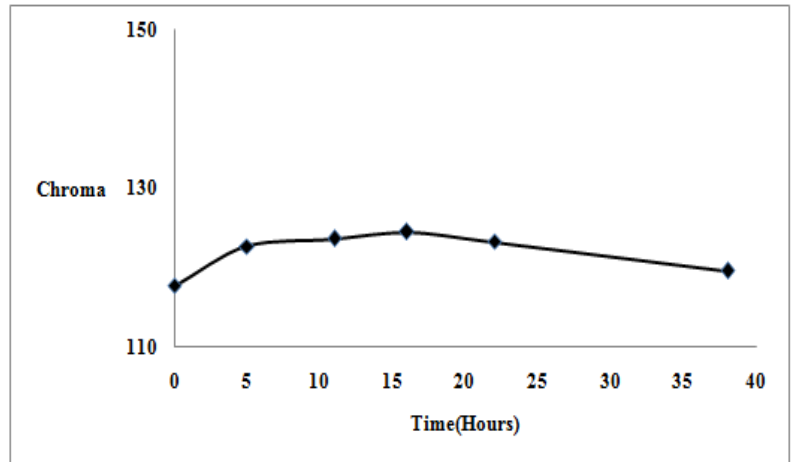

Figure 5: Changes in chroma value of Printed blister foil
The color differences are calculated using the initial spectrum for the comparison of the spectrum of exposed samples where $\Delta \mathrm{E}_{00}$ is the color difference between unexposed prints and the exposed prints with time. Figure 6 shows the color difference $\Delta \mathrm{E}_{00}$ with time. It shows that color difference is continuously increasing with time. Moreover, the visual analysis are made by the ten observers to observe the significant color change and it has been observed that the color difference is continuously increasing with variation of time due to water immersion and water spray method.

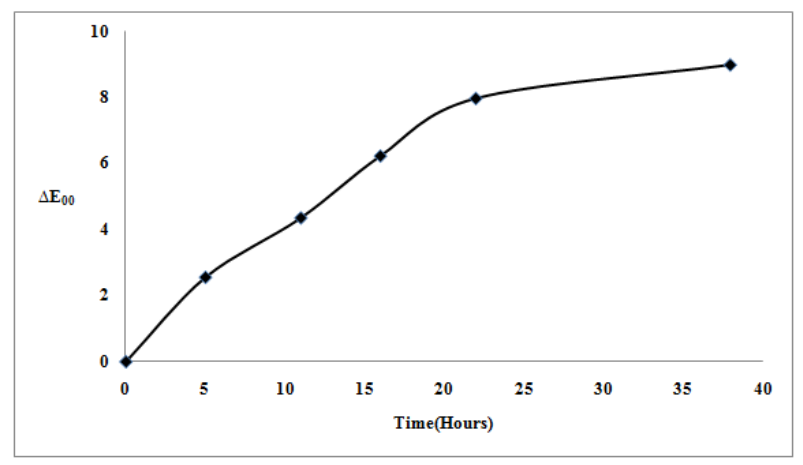

Figure 6: Color difference of printed blister foil after exposing to water

\subsection{Artificial Neural Network Performance}

In the proposed ANN model, the best performance has been obtained with 10 neurons in the hidden layer. This ANN model has been built with 5 datasets obtained from experiments to predict the repeatability of waterfastness performance of blister foil print packages over the time. In the investigation, the output (predicted) data of ANN is compared with the experimental data in Figure 7. The proposed neural network topology has been trained best with MSE value 1.2409 .

The same data used in ANN model are applied to establish the regression model in order to compare the prediction performance of regression with ANN model. Figure 7 shows the predicted reflectance for 11hours exposure using ANN model and regression model. It is observed that the prediction performance of ANN model is better than regression model. The correlation coefficient is used to check the predictive performance of the both models where it is visible that least square model and ANN model achieve 0.93 and 0.99 respectively.

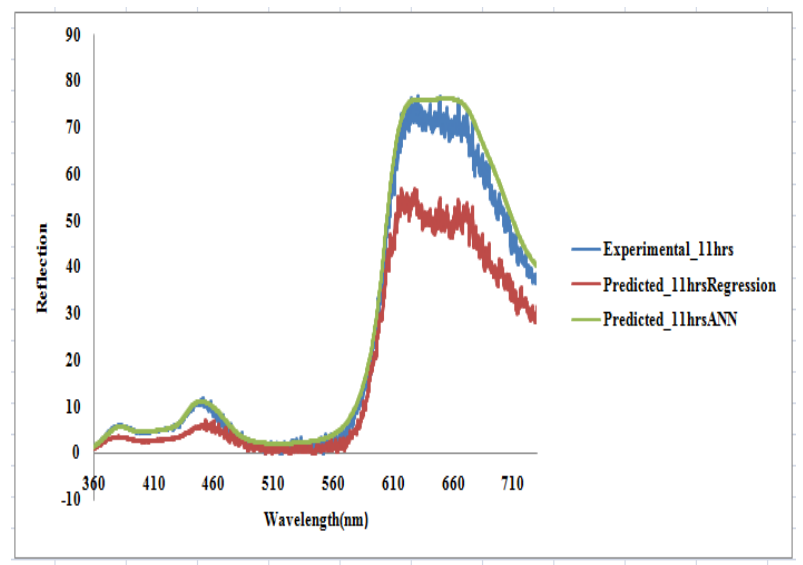

Figure 7: Experimental Value Vs Predicted Value after11 hours accelerated exposure by ANN model and Regression model 


\subsection{Discussion}

The waterfastness characteristics of prints are greatly influenced by the colorant molecules. The gravure foil inks are consisted of the proper proportion of pigment/dye, resin, solvent and additives. In the present study, the magenta foil ink is formulated with the rhodamine dye. It may be noted that the conventional rhodamine dye have poor fastness as it includes the chlorides or sulfates which are easily soluble not only in alcohols and glycols but also in water[34]. Therefore it may be suggested to add auxiliaries such as precipitants or resins which may improve the water fastness.

The result shows that the proposed neural network modeling is a better approach for the predicting the water fastness performance of foil package prints for long time exposure into the water or rains. It may be mentioned that the effect of humidity and temperature variation is assumed to be negligible as the temperature variation during the experiment was $20 \pm 2^{\circ} \mathrm{C}$ and the humidity variation was $45 \pm 1 \%$. From the result, the color change after a certain period of exposure may be predicted.

\section{CONCLUSIONS}

The goal of this current study is to predict the waterfastness of foil samples printed by gravure printing Artificial Neural Network model has been proposed to predict the fading rate on printed foil samples due to continuous exposure in water. The three-layered ANN has been configured with one hidden layer and the input layers representing of two variables time and wavelength. The most optimal ANN model has been achieved with evaluative criteria (MSE and correlation coefficient $\mathrm{R}^{2}$ ) 1.2409 and 0.9990 .On the basis of our results, the study shows that Artificial Neural Network (ANN) model gives better prediction of fading rate than the regression model. The ANN model may be used as an alternative approach for predicting the waterfastness properties of printed foils so that one can estimate the stability of the printed image due to long-term exposure to rain or water. Moreover, the magenta foil ink is printed on the foil substrate by the gravure process and with the different time interval exposure the spectrums have been calculated to indicate the fading behavior with time so that one can define the stability of foil printing. The spectral characteristics of blue and red region of magenta ink have been significantly changed with time due to exposure to water. Other primary colors may be checked in future work to verify the waterfastness performance of the prints with time. In further work, the relative importance of ink and media on the waterfastness of foil printing will be assessed to determine the permanence properties individually and then it will help to provide a method to predict the print stability due to effect of the ink media interaction. The effect of temperature and humidity on water fastness of foil printing may also be studied.

\section{ACKNOWLEDGMENTS}

Project Packmark, funded by CEFIPRA for materials and resources is acknowledged. Sergusa Solutions Pvt Ltd is acknowledged as Industrial Partner for providing materials.

\section{REFERENCES}

[1] Vikman K., "Fastness Properties of Ink Jet Prints on Coated Papers-Part 2: Effect of Coating Polymer System on Water Fastness", Journal of Imaging Science and Technology, 47(1), pp 38-43, 2003.

[2] Vikman K. and Vuorinen T., "Water Fastness of Ink Jet Prints on Modified Conventional Coatings ", Journal of Imaging Science and Technology, Vol. 48(2), pp 138-
147, 2004.

[3] Yoldas B.E., "Design of sol-gel coating media for ink-jet printing". J. Sol-Gel. Sci.Tech. 13(1-3), pp147-152, 1998.

[4] Glittenberg D., and Voigt A., "Economic formulations for improved quality ink-jet papers". Paper Tech. 42(9), pp24-29, 2001.

[5] Bugner D. E., "Handbook of imaging materials", Marcel Dekker., New York, , pp603-628,2002.

[6] Fryberg, M., Hofmann, R., and Brugger, P. A. "Permanence of ink-jet prints: A multi-aspect affair", IS\&T's NIP13: International Conference on Digital Printing Technologies. Seattle, WA. IS\&T, USA, pp 595-599, 1997.

[7] Soleimani-gorgani A. and Pishvaei M.," Water Fast Ink Jet Print Using an Acrylic /Nano-Silver Ink ", Prog. Color Colorants Coat. pp479-83, 2011.

[8] Soleimani-Gorgani1 A.and Jalili M. "Evaluating the Effect of Reactive Dye Structure and Penetrant Type on the Fastness of Ink-Jet Prints", Prog. Color Colorants Coat. 7, pp73-83, 2014.

[9] Stankovská M., Gigac J., Letko M., Opálená E.," The Effect of surface sizing on paper wettability and on properties of inkjet prints", Wood Research,59(1),pp67-76,2014.

[10] Gigac J., Stankovská M., Pažitný A.," Influence of the coating formulations and base papers on inkjet printabilit "Wood Research,61(6),pp915-926,2016.

[11] Gigac J., Stankovská M., Opálená E., Pažitný A.,” The effect of pigments andbinders on inkjet print quality", Wood Research 61(2), pp215-226, 2016.

[12] Wexler A. , Latex complexes as stabilized colorant. Kodak Polychrome Graphics, USA. U.S. Pat. 6297296 B1, 2001.

[13] Kasahara K.," A New Quick-Drying, HighWaterResistant Glossy Ink Jet Paper”, Recent Progress in Ink Jet Technologies II, Chapter 6, Ink and Media, 1999.

[14] Ahmed J., Kaur A., and Shivhare U.," Color Degradation Kinetics of Spinach, Mustard Leaves, and Mixed Puree ", Journal of Food Science 1089, .67(3), pp 10881091(2002).

[15] Balci et al.," Prediction of CIELab Data and Wash Fastness of Nylon 6,6 Using Artificial Neural Network and Linear Regression Model", Fibers and Polymers, 9(2),pp $217-224,2008$.

[16] Bas D., Dudak C. F., Hakk1 Ismail B.," Modeling and optimization IV: Investigation of reaction kinetics and kinetic constants using a program in which artificial neural network (ANN) was integrated ", Journal of Food Engineering 79(4)1152-1158 (2007).

[17] Kov'acs B., T'oth J.," Estimating Reaction Rate Constants with Neural Networks" International Journal of Applied Mathematics and Computer Science ,4(1) , pp $1305-5313,2007$.

[18] Dutot A, Rude J, Aumont B.," Neural network 
method to estimate the aqueous rate constants for the $\mathrm{OH}$ reactions with organic compounds ", Atmospheric Environment 37(2),pp 269-276, 2003.

[19] Mostafa A., Mehdi N., Hassan A.," New Approach in Modeling of Metallocene-Catalyzed Olefin Polymerization Using Artificial Neural Networks", Macromol. Theory Simul., 18(3) ,pp195-200,2009.

[20] Fernandes N. A. and Lona. F. M, "Neural Network Application Polymerization Processes", Brazilian Journal Of Chemical Engineering, . 22(3) ,pp $401-418,2005$.

[21] Kuroda C., Kim J., "Neural network modeling of temperature behavior in an exothermic polymerization process", Neurocomputing ,43(1-4),pp 77-89,2002.

[22] Christo C. F., Masri R. A., Nebot M. E. , "Utilising artificial neural network and repro-modeling in turbulent combustion", Proceedings of the IEEE International Conference on Neural Networks, Perth, 1, December, 1995.

[23] Fathi M.; Mohebbi M;Razavi Mohammad Ali S., "Application of Image Analysis and Artificial Neural Network to Predict Mass Transfer Kinetics and Color Changes of Osmotically Dehydrated Kiwifruit", Food and Bioprocess Technology, 4(8),pp1357-1366, 2011.

[24] Tang Y. S. et al," Application of Artificial Neural Network to Predict Colour Change, Shrinkage and Texture of Osmotically Dehydrated Pumpkin", IOP Conference Series: Materials Science and Engineering 206 June, 2017.

[25] Goyal S et al., "Shelflife Prediction of Processed Cheese Using Artificial Intelligence ANN Technique", Croatian Journal of Food Technology, Biotechnology and Nutrition ,7 (3-4), pp184-187 (2012).
[26] Goyal S, Goyal G. K. "Development of intelligent computing expert system models for shelf life prediction of soft mouth melting milk cakes". International Journal of Computer Applications, 25(9), 41-44, 2011.

[27] Mandal M. and Bandyopadhyay S. "To predict the lightfastness rate of foil prints To Predict The Lightfastness Rate of Foil Print Applying Artificial Neural Network" Communicated to Packag Technol Sci. June 2019.

[28] Basheer I.A. and Hajmeer M.," Artificial neural networks: fundamentals, computing, design, and application", Journal of Microbiological Methods, 43 ,pp3-31,2000.

[29] Haykin, S. Neural networks: a comprehensive foundation. NewJersey: Prentice Hall. (1994).

[30] Sharma G., Edul W.Wu, Dalal N," The CIEDE2000 Color-Difference Formula: Implementation Notes, Supplementary Test Data, and Mathematical Observations ", COLOR research and application, 30(1) ,pp 21-30 (2005).

[31] Marsland S.. Machine Learning: An Algorithm Perspective.New York: Chapman and Hall/CRC.(2009).

[32] Bealen M.H., Hagan M.T.,and Demuth H.B., Neural Network Toolbox, Revised for Version 7.0 (Release 2010b), 2010.

[33] Wilhelm H and McCormick-Goodhart M., "An overview of the permanence of inkjet prints with Traditional color prints", The Society of Imaging Science and Technology, 2000 .

[34] Andree F et al. "Rhodamne Dyes Which Are Sparingly Soluble Or Insoluble $\mathrm{N}$ Water" USA. U.S. Pat. 3,708,499, 1973. 